\title{
Role of Biologics in the Development of Autoimmune Hepatitis: A Review
}

\author{
Leon D. Averbukh* and George Y. Wu \\ Department of Medicine, Division of Gastroenterology-Hepatology, University of Connecticut Health Center, Farmington, CT, USA
}

\begin{abstract}
Autoimmune hepatitis (AIH) is a cause of chronic, immunemediated liver injury which without treatment may progress to end-stage liver disease. The disease state, characterized by elevations in liver enzymes, autoantibodies, and interface hepatitis on histology, has been noted to be induced by a wide range of insults. Medications, most commonly minocycline and nitrofurantoin, have long been established as potential inducers of AIH. Recently, biologics, powerful immune-modulators, have also been reported to induce AIH. We conclude that there is an association between administration of biologics in the development of AIH, and whether the relationship is causal will require appropriate studies in the future.
\end{abstract}

Citation of this article: Averbukh LD, Wu GY. Role of Biologics in the Development of Autoimmune Hepatitis: A Review. J Clin Transl Hepatol 2018;6(4):402-409. doi: 10.14218/JCTH.2018.00039.

\section{Introduction}

Autoimmune hepatitis (AIH) is a chronic illness of the liver characterized by hepatocellular inflammation and necrosis, with interface hepatitis and plasma infiltration of liver tissue. ${ }^{1}$ On laboratory evaluation, autoantibodies, particularly antinuclear antibodies (ANA), smooth muscle antibodies (SMA), liver kidney microsome type 1 antibodies and serum immunoglobulin (Ig)G, are often found to be abnormally increased. ${ }^{2}$ Aminotransferase levels can be mild or severely elevated. Cases of AIH can be asymptomatic or present with signs of acute liver disease, chronic liver disease or, even fulminant hepatic failure. ${ }^{3}$ Symptoms of the disease are frequently nonspecific and include fatigue, lethargy, malaise, anorexia, nausea, abdominal pain, itching, and arthralgia involving the small joints. ${ }^{4}$

The disease is known to affect women more commonly than men ( $70-80 \%$ of cases involving females) and age pattern shows a preponderance for those in their $4^{\text {th }}$ to $6^{\text {th }}$ decades of life. ${ }^{5} \mathrm{AIH}$ is most prevalent in European populations, with rates

Keywords: Autoimmune hepatitis; TNF alpha inhibitors; Biologics; Hepatitis; Infliximab; Adalimumab; Etanercept.

Abbreviations: AIH, autoimmune hepatitis; ANA, antinuclear antibodies; DILI, drug-induced liver injury; ds, double-stranded; IAIHG, International Autoimmune Hepatitis Group; Ig, immunoglobulin; SMA, smooth muscle antibodies; TNF $\alpha_{\text {, }}$ tumor necrosis factor alpha.

Received: 25 June 2018; Revised: 9 August 2018; Accepted: 13 August 2018

$*$ Correspondence to: Leon D. Averbukh, Department of Medicine, Division of Gastroenterology-Hepatology, University of Connecticut Health Center, 236 Farmington Ave., Farmington, CT 06030, USA. E-mail: averbukh@uchc.edu of the disease in Caucasian populations of Europe and North America ranging from 0.1 to $1.9 / 100,000 /$ year. $^{5}$ Considerably less frequently, the disease has also been observed in Japan. ${ }^{6}$ Unfortunately, the data on the disease prevalence in other regions of the world are very limited, and a lack of universally accepted diagnostic methods has resulted in reported prevalence rates that over- or under-estimate the true prevalence.

Several diagnostic criteria have been developed to identify $\mathrm{AIH}$. The most commonly used at this time are the diagnostic criteria of the International Autoimmune Hepatitis Group (IAIHG), revised original diagnostic scoring system of the IAIHG, and the simplified diagnostic scoring system of the IAIHG. Some scoring systems, such as the simplified scoring system of the IAIHG, are designed to be basic enough that they can be easily employed in the clinical setting, while others are meant to discern more challenging cases. ${ }^{7}$ The central theme of all these devised scoring systems is that clinical judgement remains an important aspect of diagnosis and that liver biopsy is required. Standard therapy, used to help slow the disease progression and resolve symptoms, consists of immunosuppressives, steroids with or without the addition of purine analogs, azathioprine, or 6-mercaptopurine. Because of the relatively specific response of autoimmune conditions to immunosuppressives, positive responses have been incorporated into some of the diagnostic scoring systems for AIH.

The two scoring systems used in the cases reviewed currently were the Revised Original Scoring System of the International Autoimmune Hepatitis Group and the Simplified Diagnostic Scoring System of the International Autoimmune Hepatitis Group. 7,8

The causes and pathogenesis of AIH are not completely understood but likely involve both environmental and genetic factors. The common theme among all potential factors is the abnormal stimulation of the host immune system. With regards to medication-induced $\mathrm{AIH}$, it is important to distinguish between preexisting subclinical AIH made clinically apparent by exposure to medications versus drug-associated development of AIH de novo in patients with no prior evidence of AIH. In an effort to clarify the terminology, the implementation of a three-category system has been suggested. ${ }^{9}$

The first of the three categories that they describe is referred to as "AIH with DILI", where DILI stands for druginduced liver injury. In these cases, patients already have documented AIH along with possible advanced fibrosis on histology at the time of exposure to a drug, with subsequent liver injury. ${ }^{9}$

The second category is "drug-induced AIH". In this category are patients who probably have subclinical AIH and are unaware and undiagnosed or those that have a predisposition 
for AIH. It is proposed that DILI unmasks or triggers the condition. This group is considered to have AIH and discontinuation of the offending agent alone does not result in resolution of the inflammation. Cessation of immunosuppressive therapy is frequently associated with relapse of inflammation. ${ }^{9}$

The third category is the "immune-mediated DILI" group. For these patients, there are no signs or symptoms of AIH prior to medication exposure. Typical characteristics of symptoms and markers of liver damage and abnormal serology are absent and appear only after exposure to an offending medication. Patients in this category show good response to steroid therapy and importantly, often achieve long-term remission after withdrawal of immunosuppressives. It seems possible that simple cessation of the offending medication may be sufficient in mild cases. ${ }^{9}$ While AIH and immune-mediated DILI carry different long-term prognoses, the pathogenic mechanisms between them appear to be similar. Both disease states result in an immune-mediated hepatitis and are triggered or evolve from an initial insult brought on by the addition of a medication. For this reason, they are described in a similar manner in this review.

With regards to treatment, some experts have recommended that treatment be initiated for any diagnosis of $\mathrm{AIH}$, while others have advised therapy for only certain criteria of liver damage. ${ }^{8,10}$ In this review, we examine the evidence for the association of biologics (infliximab, adalimumab, and etanercept) in the development of $\mathrm{AIH}$ with an emphasis on category 3, de novo biologic-induced, immune-mediated hepatitis. We also review proposed pathogenic mechanisms, diagnoses, and treatment.

\section{Monoclonal antibody therapy: Tumor necrosis factor alpha $(T N F \alpha)$ inhibitors}

Biologics include a broad range of products whose production involves recombinant DNA technology. This broad category of medications is generally further subdivided into three groups, as follows: agents that are nearly identical to the body's own signaling proteins; fusion proteins; and, monoclonal antibodies. The first subset-substances identical to the body's own signaling proteins-includes stimulating factors such as erythropoietin, growth hormone, and biosynthetic human insulin. The second subset, known as the fusion proteins, involves the combination of a naturally occurring receptor linked to an Ig frame. The resulting structure contains an Ig and a corresponding receptor necessary for the protein's specificity. The third category-monoclonal antibodies created using techniques such as hybridoma or monoclonal antibody technology-consists of custom designed antibodies, the purpose of which is to target a specific cell type and either stimulate or inhibit the immune system, or target radioactivity to certain cells.

Monoclonal antibodies are further subdivided into three main types, as follows: recombinant; chimeric; and, human. Recombinant monoclonal antibodies involve the production of antibodies using yeast or viruses. Chimeric monoclonal antibodies involve the production of humanized DNA by combining portions of mouse and human antibodies by recombinant technology. The final category-human antibodies-is the most recent development in monoclonal antibody technology and involves the generation of fully human monoclonal antibodies by either the use of transgenic mice or through phage display in which variable antibody domains are displayed on filamentous phage coat proteins.

While monoclonal antibodies have been designed for a wide array of molecular targets, in this review, we specifically examine TNF $\alpha$ inhibitors. This type of monoclonal antibody is designed to inhibit the proinflammatory actions of TNF $\alpha$ Some of the most potent immune inhibitors and agents in this class are infliximab, adalimumab, and etanercept. Infliximab is a chimeric human-mouse IgG monoclonal antibody derived from recombinant DNA that consists of mouse heavy and light chain variable regions combined with human heavy and light chain constant regions. ${ }^{11}$ Adalimumab on the other hand, is a fully human monoclonal antibody, while etanercept, is a TNF receptor-IgG fusion protein. ${ }^{12,13}$

TNF $\alpha$ inhibitors are used to treat autoimmune conditions, including ulcerative colitis, ankylosing spondylitis, Crohn's colitis, psoriasis, hidradenitis suppurativa and rheumatoid arthritis. ${ }^{14-29}$ Their mechanism of action involves binding and inhibiting $\mathrm{TNF} \alpha$, a major inflammatory signal protein. ${ }^{30}$ Unfortunately, due to the severe immunosuppression that these agents can cause, immune defenses can be compromised, resulting in serious bacterial infections, such as tuberculosis, reactivation of hepatitis $B$, and hepatosplenic T-cell lymphoma. ${ }^{31,32}$ Moreover, despite inducing immune suppression, TNF $\alpha$ inhibitors have been implicated in the development of other autoimmune diseases, including drug-induced lupus, demyelinating central nervous system disorders, psoriasis and psoriasiform skin lesions, and new-onset vitiligo. ${ }^{33}$ $\mathrm{AIH}$ has also been a reported outcome of TNF $\alpha$ inhibitor use, although the evidence has been limited to case reports, a summary of which is listed in Table 1.

\section{Infliximab}

As seen in Table 1, females experienced a predilection for disease state, as 13 of the patients were female, while only 6 were male. None of the reported cases had a history of chronic or serious liver disease (although 3 had evidence of chronic hepatitis on biopsy). All reported patients had significantly elevated aminotransferases (aspartate aminotransferase, alanine aminotransferase), 16 patients were reported to have significantly elevated ANA titers, 6 had significantly elevated SMA titers, 8 had significantly elevated doublestranded (ds)DNA titers, and 8 had significantly elevated IgG titers. Other than 1 patient with significant ANA titers prior to treatment administration, none of the cases reported significant titer elevations prior to infliximab initiation. ${ }^{14}$ All 19 cases reviewed here reported liver biopsies in their results and all had evidence of interface hepatitis of varying degrees of severity. The average time to onset of disease post-medication application ranged from a couple to several months, with the fastest reported time to onset being within 2 weeks of therapy initiation and the longest being 2 years. Seventeen of the nineteen cases had resolution of aminotransferase elevations following cessation of steroids (although three had lingering ANA and one had persistently increased gamma-glutamyltransferase). Two of the cases required long-term therapy with corticosteroids for treatment.

Using the previously described categorization, it appears that most of the patient cases fall into the $3^{\text {rd }}$ categoryimmune-mediated DILI. This is based on complete resolution of AIH symptoms and decreasing titer levels (ANA, SMA, IgG, anti-dsDNA) with immunosuppressives. However, there was 
Averbukh L.D. et al: Biologics in AIH: A review

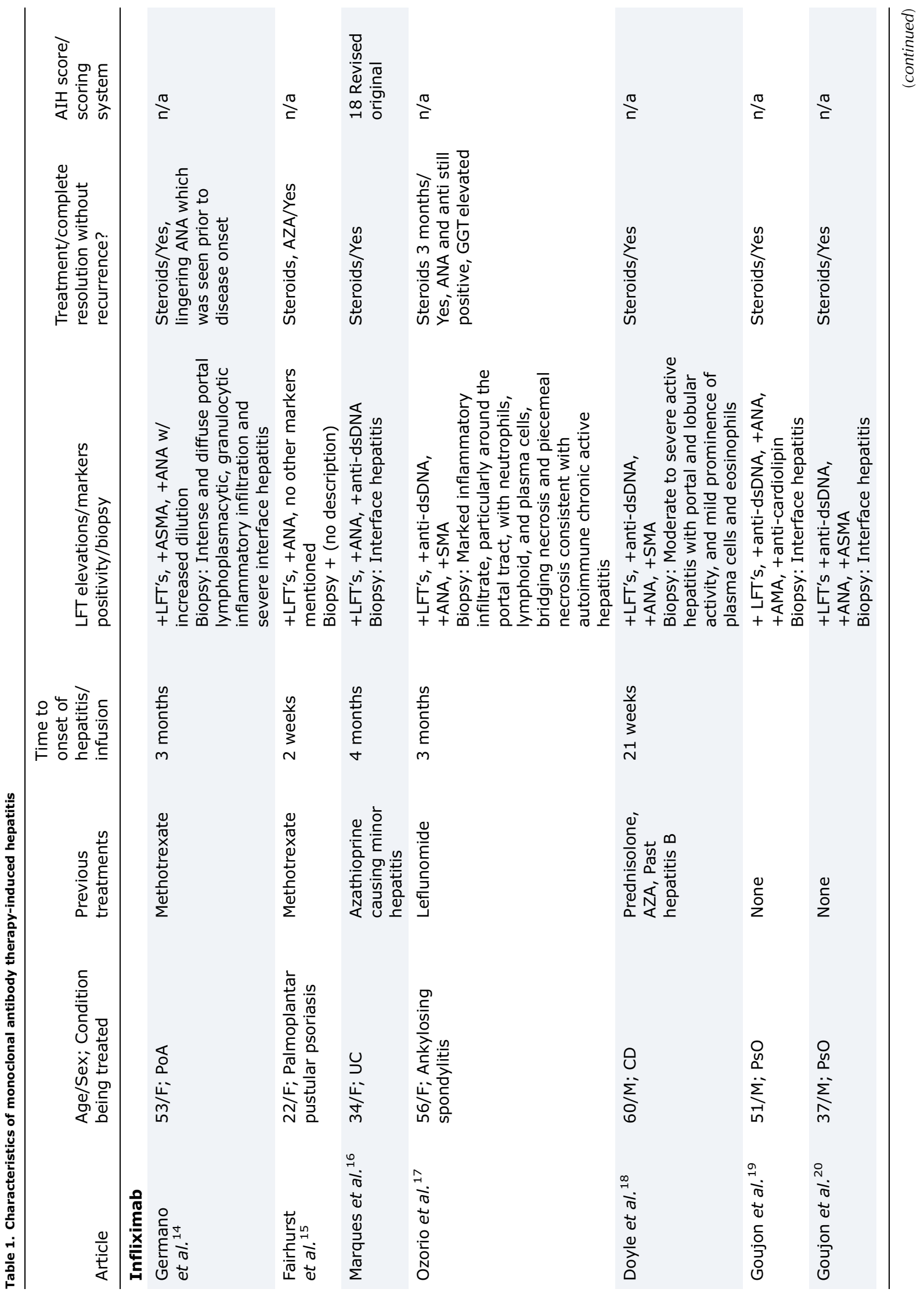




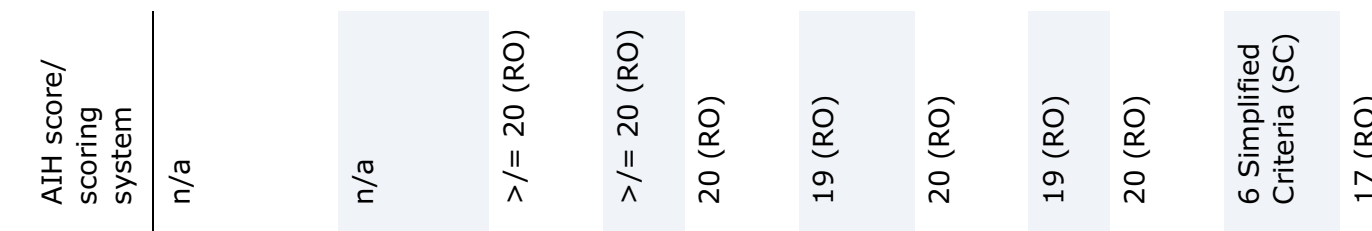
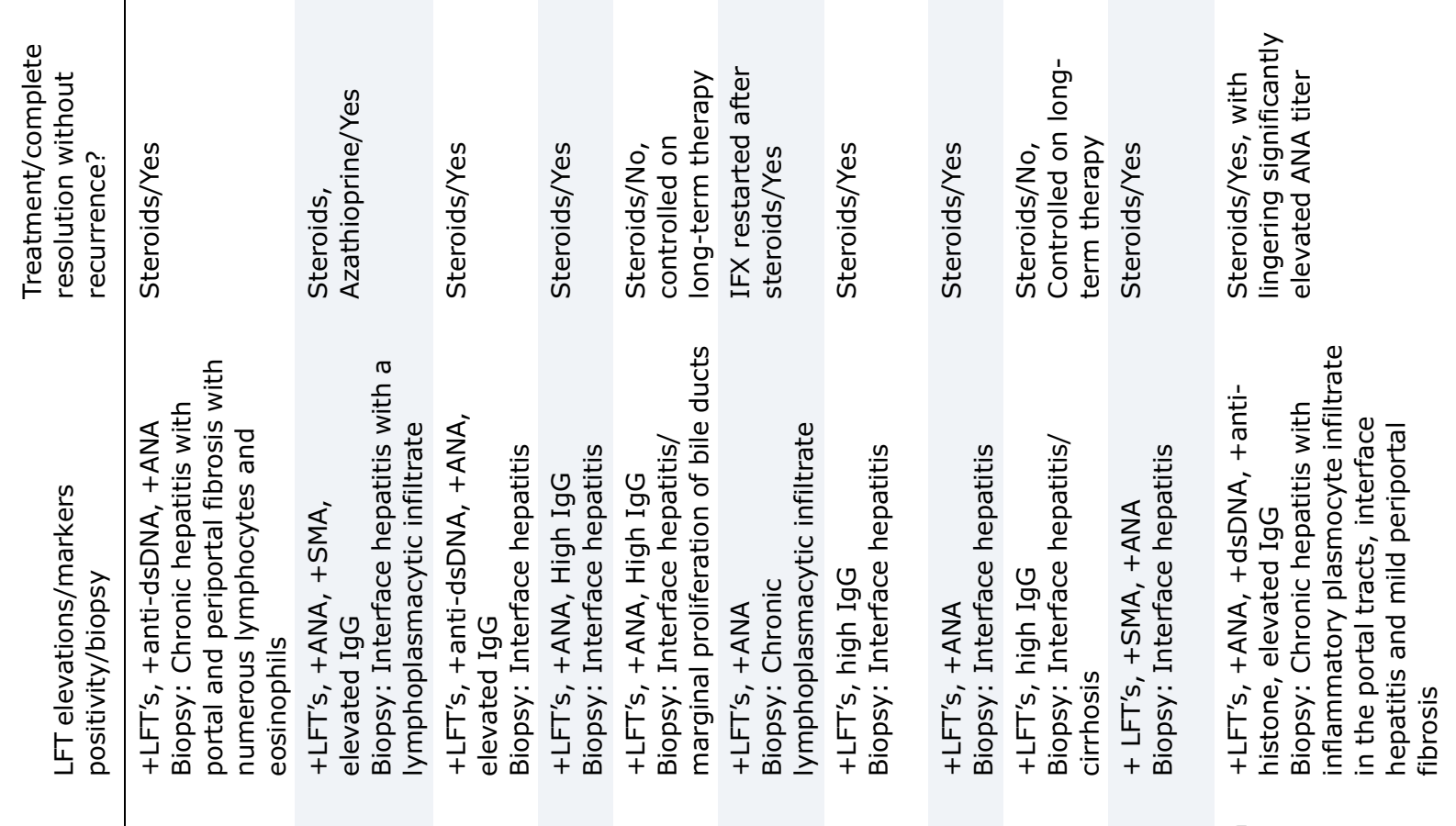

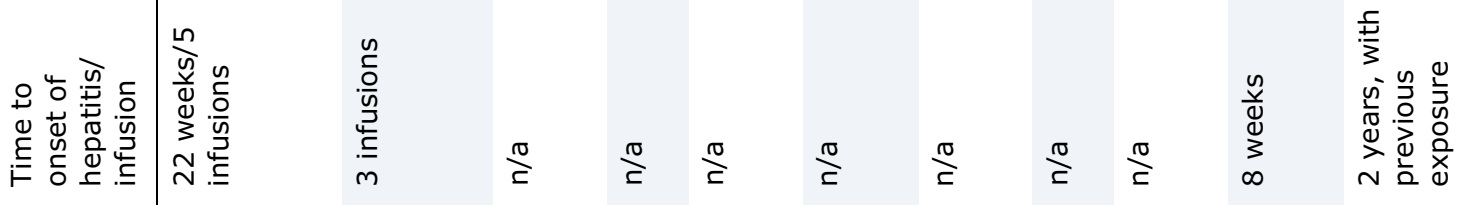
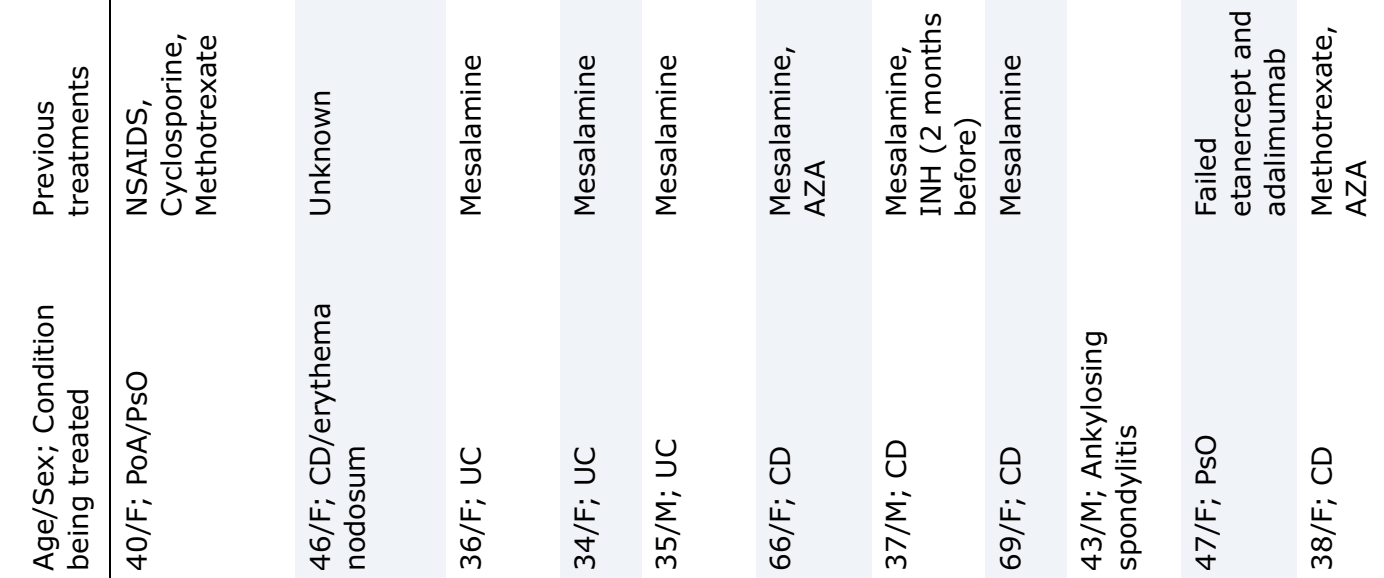

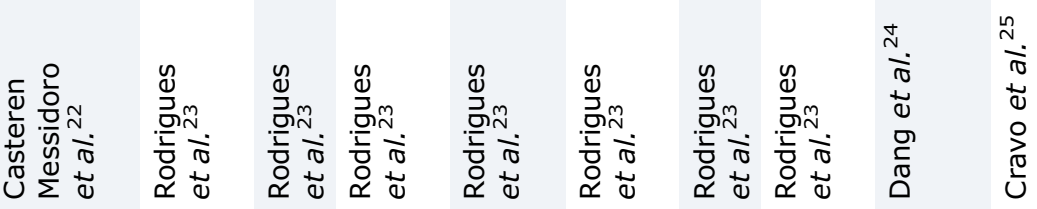


Averbukh L.D. et al: Biologics in AIH: A review

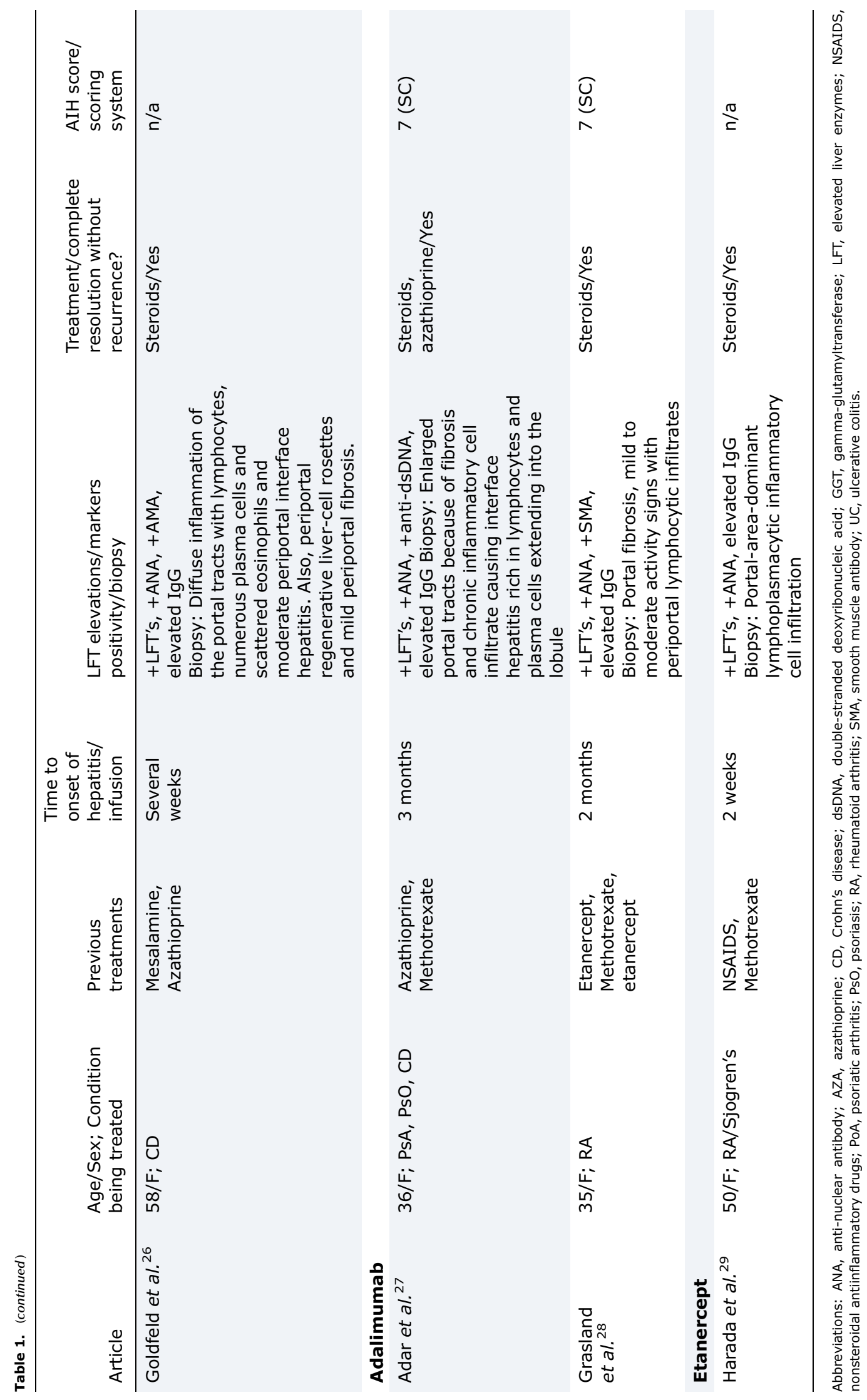


no description of long-term follow-up in the cases reviewed, making it possible that patients relapsed after the reported studies were completed. Furthermore, none of the cases involved simply stopping the offending agent as a suggested method of identifying immune-mediated DILI. Infliximab was stopped in all cases, but none of the cases relied on cessation of agent alone to achieve resolution, presumably because of the severity of reactions. All of the cases responded to steroid treatment.

Two other cases required long-term steroid maintenance therapy. These may represent examples of true drug-induced $\mathrm{AIH}$ with possible unmasking of AIH by infliximab. Unfortunately, there were no preinfliximab administration liver biopsies taken on those patients to further clarify whether there was a preexisting subclinical $\mathrm{AIH}$, making this diagnosis speculative. ${ }^{23}$

Of note, one case involving infliximab therapy showed resolved hepatitis poststeroid therapy; although, an elevated ANA, with no documented titer, remained. ${ }^{14}$ Although the ANA titer prior to infliximab therapy was elevated at 1:80, the low level, lack of specificity of ANA, and lack of biopsy prior to infliximab therapy to diagnose a preexisting $A I H$, led to insufficient evidence to qualify this as a case of druginduced $\mathrm{AIH}$.

None of the papers in this review fit into the categorization of AIH with DILI by its strict definition, as none of the patients were reported to have known AIH prior to starting their respective monoclonal antibody therapies. However, 5 cases did exhibit evidence of chronic liver disease on biopsy, although only 3 are discussed below as the 2 others did not provide case descriptions. ${ }^{17,21,23,25}$

A case with computed tomography findings of nonhomogeneous liver texture and biopsy showing inflammatory infiltrate around the portal tract with neutrophils, lymphoid and plasma cells, as well as bridging necrosis and piecemeal necrosis consistent with autoimmune chronic active hepatitis, has been reported. ${ }^{17}$ Prior to initiation of infliximab, normal serology and negative ANA titers were reported. For this reason, we placed that case in the drug-induced AIH category; moreover, a standard course of steroid therapy did not lead to resolution, and the patient continued to have significantly elevated IgG, ANA and dsDNA antibody, and positive SMA. It is unclear what alternative therapy was employed after treatment failure.

One case demonstrated chronic hepatitis with portal and periportal fibrosis with numerous lymphocytes and eosinophils on biopsy after discontinuation of infliximab. Unlike the other cases in this review, this one provided the results of biopsy prior to initiation of infliximab, as the patient had experienced enzyme elevations while on methotrexate (which was subsequently discontinued with normalization of lab values prior to infliximab initiation). The preceding biopsy showed only mild, nonspecific changes. ${ }^{21}$ However, that case also had a complicating diagnosis of lupus erythematosus. As the pre- and during infliximab treatment biopsies were taken roughly 2 years apart, it is possible that the portal and periportal fibrosis was the culmination of multiple hepatic insults, including a smoldering lupus, on a liver that was already inflamed from previous treatment modalities. This case was categorized as immune-mediated DILI because steroid therapy successfully led to resolution of symptoms and there was not enough evidence to diagnose a pre-existing $\mathrm{AIH}$. Of note, we considered lupus hepatitis as an alternative primary cause of liver dysfunction rather than immune mediated DILI in this patient since the two are often difficult to discern on serology and have significant overlap symptomatically. While the serologies provided in this case did not strongly advocate for one etiology of liver disease over the other, the patient's biopsy was more phenotypically consistent with AIH rather than lupus hepatitis, as periportal fibrosis, rather than lobular fibrosis, was evident. 34

In another case, a biopsy of the liver showed inflammatory plasmocyte infiltrates in the portal tracts, interface hepatitis, and mild periportal fibrosis. ${ }^{25}$ Again, confounding variables complicated the diagnosis of drug-induced AIH. The patient described in the case had undergone infliximab therapy on three separate occasions within the past decade. While the patient did not experience significant hepatitis during previous drug administrations, the hepatic fibrosis seen on biopsy may have been the result of previous DILI secondary to infliximab therapy. That case was also classified as an immune-mediated DILI, as there was no convincing evidence of longstanding AIH.

Three of the cases reviewed showed surprising responses to re-initiation of TNF $\alpha$ inhibitor therapy after resolution of acute AIH-like symptoms post-infliximab treatment. In one case, a 66 year-old female with Crohn's colitis was cautiously restarted on infliximab and tolerated the continued treatment without any further reported episodes of liver injury (though how this was monitored is not stated in the report). ${ }^{23}$ In two cases, patients were switched to adalimumab, another TNF $\alpha$ inhibitor, after symptom resolution and decrease in enzyme levels. ${ }^{25,26}$ In one case, only ANA titers remained significantly elevated at 1:160-1:320 with adalimumab use, while in the other report the patient had no positive serologies after starting adalimumab. Neither case provided any further liver biopsy data.

The re-initiation of TNF $\alpha$ inhibitors without further hepatitis flares complicates the relationship between TNF $\alpha$ inhibitors and the development of AIH. One of the proposed theories as to why infliximab appears to be more commonly associated with AIH-like syndromes as opposed to the other TNF $\alpha$ inhibitors may lie in the molecular make-up of these medications; infliximab is a chimeric human-mouse IgG monoclonal antibody, while adalimumab is fully humanized and etanercept is a fusion of recombinant soluble TNF $\alpha$ receptor and human IgG monoclonal antibody. It is believed that the murine portion of infliximab may make it more susceptible to causing immune-mediated reactions. ${ }^{35}$

This theory, however, does not explain why a patient reacted to infliximab during initial therapy yet tolerated reintroduction of the medication once their acute hepatitis resolved nor does it explain why, in another case, the patient previously tolerated infliximab therapy on multiple occasions without experiencing symptomatic hepatitis. ${ }^{23,24}$ This may indicate that even on an individual level, susceptibility might have less to do with predisposition and more to do with circumstance.

\section{Adalimumab}

As seen in Table 1, there were currently only two reported cases of adalimumab associated with AIH disease. In those cases, both patients exhibited AIH symptoms and were females under the age of 45 who did not have any positive serological markers for AIH prior to adalimumab administration. For both patients, AIH symptoms developed within months of initiation of treatment with adalimumab and liver 
biopsies were consistent with AIH. Fortunately, both patients had resolution of their hepatitis and normalization of aminotransferases after steroid treatment, supporting a diagnosis of immune-mediated DILI. Interestingly, one of the patients had been treated in the past with etanercept, which has also been reported to induce $\mathrm{AIH}$ and hepatic fibrosis.

\section{Etanercept}

As seen in Table 1, literature review yielded only a single reported case of etanercept causing a suspected AIH. The patient was a female, over 45 years-old, with RA and Sjogren's syndrome, and who, after being unsuccessfully treated with nonsteroidal anti-inflammatory drugs and methotrexate, was started on a course of etanercept. Two weeks after starting etanercept, she developed elevated liver enzymes with ANA positivity, significantly elevated IgG titers, and biopsy proven portal area-dominant lymphoplasmacytic inflammatory cell infiltration. The patient responded well to a course of steroid treatment and achieved complete resolution. Of note, the patient did have a previous reported hepatitis, thought to be secondary to nonsteroidal antiinflammatory drug use, which was evidenced by mild liver enzyme elevations of aspartate aminotransferase (45 U/L) and alanine aminotransferase $(62 \mathrm{U} / \mathrm{L})$. All other tests for serological markers prior to etanercept initiation were negative.

In this case, the authors initially postulated that the patient had mild liver disease with liver enzyme elevation secondary to nonsteroidal anti-inflammatory drug use, though on reevaluation believed that this may have been an undiagnosed $\mathrm{AIH}$ which was exacerbated with etanercept therapy. ${ }^{29}$ As the patient had a resolution of titers and normalization of enzyme levels after steroid course with no long-term follow-up, it is not possible to determine whether this was a missed AIH with exacerbation or if it was an autoimmune-induced DILI overlying previous DILI secondary to nonsteroidal anti-inflammatory drug use.

\section{Mechanisms behind biologic-triggered AIH and AIH-} like symptoms

It is unclear why, and seems counter-intuitive that, biologics, such as infliximab and other TNF $\alpha$ inhibitors, which are known to be such powerful immunosuppressives, can induce AIH. Generally, AIH is thought to be caused by a loss of tolerance against the liver, presenting as a periportal hepatitis initiated by $\mathrm{CD} 4+\mathrm{T}$ cells which recognize selfantigens. ${ }^{36}$ As the hepatocytes express aberrant HLA class II molecules on their surface, resultant inflammatory processes occur with the destruction of hepatocytes by cellmediated autoimmunity. ${ }^{36,37}$ Alternatively, the induction of AIH may be the result of antibody-dependent autoimmunity, with antibodies directed against hepatocyte membranes. These autoantibodies include IgG, which is found in $80 \%$ of patients with $\mathrm{AIH}{ }^{38}$

Monoclonal antibodies are hypothesized to contribute to the development of immune-mediated liver disease through the development of autoantibodies, including ANA and antidsDNA, in predisposed patients. One proposed pathway involves TNF $\alpha$ agents interfering with induced cell death of CD8 T cells, leading to an increased lymphocyte presence. ${ }^{39}$ Alternatively, TNF $\alpha$ blockade could interfere with the normal cytotoxic T lymphocyte suppression of self-reactive B cell pro- duction. ${ }^{39}$ Another potential cause of AIH with monoclonal antibodies may have less to do with the activation of complex pathways and more with drug-induced hepatic cell injury exposing antigens which normally have no contact with immune cells. The exposed antigens are subsequently attacked by immune cells, leading to liver damage. ${ }^{40}$

Interestingly, the frequency of autoimmune reactions is greater when TNF $\alpha$ inhibitors are used as treatment for specific rheumatological diseases, and when the medications are used in combination with other agents such as methotrexate. ${ }^{17}$

\section{Limitations in studies on medication-induced AIH}

AIH is difficult to define due to its lack of disease-defining features and potential causes. Although there are scoring systems designed to make a diagnosis of drug-induced liver disease, these scoring systems, such as the Roussel Uclaf Causality Assessment Method, have been found to be too burdensome for regular clinical use. Other scales, less focused on medication-induced liver disease and more so on identifying true AIH in the clinical setting, are the International Diagnostic Criteria for AIH Scoring System as well as the more recent simplified version. ${ }^{39,7}$

Unfortunately, even with the scoring systems in place, it is difficult to prove AIH. In fact, the cases in this review that employed an AIH scoring system identified scores correlating to a "high likelihood" for AIH. Still, uniform use of classification systems is important for disease understanding, especially in cases of possible medication-induced disease. Of the 22 case reports examined in this review, only 12 calculated scores for the drug-induced disease state (Simplified Criteria, International Diagnostic Criteria for AIH Scoring System). ${ }^{16,22-24,26,27}$ Furthermore, none of the authors contributed AIH scores post-cessation of steroid therapy, likely because most patients saw resolution of symptoms. A standardized scoring approach with scores prior to treatment, during treatment with therapeutic agents, and posttreatment with steroid therapy would be helpful to further understand $\mathrm{AIH}$.

A significant confounding variable in the attempt to draw causation between certain agents and the development of AIH-like symptoms is the nature of treatment histories. As seen in this review, patients who received monoclonal antibody therapy had previously undergone many other failed therapies with agents that themselves have toxic hepatic safety profiles, such as methotrexate, leflunomide, and mesalamine. In addition to the effect of confounding variables is the fact that in some of the cases reviewed, reintroduction of the agents initially blamed for the AIH symptoms or use of biologics with the same mechanism of action did not lead to any further reported acute hepatitis events.

\section{Conclusions}

Biologics, in particular TNF $\alpha$ inhibitors, are an important and increasingly used therapy for autoimmune disorders. It appears, however, that they may paradoxically potentiate or cause AIH and variants of similar pathogenic mechanisms, such as immune-mediated DILI. Although the data are sparse and there are no large or controlled studies on the subject, the cases reviewed do suggest an association. It will be important to engage in larger studies to establish the cause 
and effect relationship between biologics and their role in inducing AIH and similar disease processes.

\section{Conflict of interest}

The authors have no conflict of interests related to this publication.

\section{Author contributions}

Writing of the manuscript (LDA), development of the idea for the article, and critically revised the manuscript (GYW).

\section{References}

[1] Czaja AJ. Drug-induced autoimmune-like hepatitis. Dig Dis Sci 2011;56: 958-976. doi: 10.1007/s10620-011-1611-4.

[2] Czaja AJ. Performance parameters of the conventional serological markers for autoimmune hepatitis. Dig Dis Sci 2011;56:545-554. doi: 10.1007/ s10620-010-1501-1.

[3] Krawitt EL. Autoimmune hepatitis: classification, heterogeneity, and treatment. Am J Med 1994;96:23S-26S doi: 10.1016/0002-9343(94)90186-4.

[4] Krawitt EL. Clinical features and management of autoimmune hepatitis. World J Gastroenterol 2008;14:3301-3305 doi: 10.3748/wjg.14.3301.

[5] Ngu JH, Bechly K, Chapman BA, Burt MJ, Barclay ML, Gearry RB, et al. Population-based epidemiology study of autoimmune hepatitis: a disease of older women? J Gastroenterol Hepatol 2010;25:1681-1686. doi: 10. $1111 / j .1440-1746.2010 .06384 . x$.

[6] Boberg KM. Prevalence and epidemiology of autoimmune hepatitis. Clin Liver Dis 2002;6:635-647 doi: 10.1016/S1089-3261(02)00021-1.

[7] Czaja AJ. Diagnosis and management of autoimmune hepatitis: Current status and future directions. Gut Liver 2016;10:177-203. doi: 10.5009/gnl15352.

[8] Manns MP, Czaja AJ, Gorham JD, Krawitt EL, Mieli-Vergani G, Vergani D, et al. Diagnosis and management of autoimmune hepatitis. Hepatology 2010;51: 2193-2213. doi: 10.1002/hep. 23584.

[9] Weiler-Normann C, Schramm C. Drug induced liver injury and its relationship to autoimmune hepatitis. J Hepatol 2011;55:747-749. doi: 10.1016/j.jhep. 2011.02.024.

[10] Liwinski T, Schramm C. Autoimmune hepatitis - update on clinical management in 2017. Clin Res Hepatol Gastroenterol 2017;41:617-625. doi: 10. 1016/j.clinre.2017.07.002.

[11] Akiho H, Yokoyama A, Abe S, Nakazono Y, Murakami M, Otsuka Y, et al. Promising biological therapies for ulcerative colitis: A review of the literature. World J Gastrointest Pathophysiol 2015;6:219-227. doi: 10.4291/wjgp.v6.i4.219.

[12] Brekke $\mathrm{OH}$, Sandlie I. Therapeutic antibodies for human diseases at the dawn of the twenty-first century. Nat Rev Drug Discov 2003;2:52-62. doi: $10.1038 / \mathrm{nrd} 984$.

[13] Zalevsky J, Secher T, Ezhevsky SA, Janot L, Steed PM, O'Brien C, et al. Dominant-negative inhibitors of soluble TNF attenuate experimental arthritis without suppressing innate immunity to infection. J Immunol 2007;179: 1872-1883 doi: 10.4049/jimmunol.179.3.1872.

[14] Germano V, Picchianti Diamanti A, Baccano G, Natale E, Onetti Muda A, Priori $\mathrm{R}$, et al. Autoimmune hepatitis associated with infliximab in a patient with psoriatic arthritis. Ann Rheum Dis 2005;64:1519-1520. doi: 10.1136/ard. 2004.032821.

[15] Fairhurst DA, Sheehan-Dare R. Autoimmune hepatitis associated with infliximab in a patient with palmoplantar pustular psoriaisis. Clin Exp Dermatol 2009;34:421-422. doi: 10.1111/j.1365-2230.2008.03088.x.

[16] Marques M, Magro F, Cardoso $\mathrm{H}$, Carneiro F, Portugal R, Lopes J, et al. Infliximab-induced lupus-like syndrome associated with autoimmune hepatitis. Inflamm Bowel Dis 2008;14:723-725. doi: 10.1002/ibd.20293.

[17] Ozorio G, McGarity B, Bak H, Jordan AS, Lau H, Marshall C. Autoimmune hepatitis following infliximab therapy for ankylosing spondylitis. Med J Aust 2007; 187:524-526.

[18] Doyle A, Forbes G, Kontorinis N. Autoimmune hepatitis during infliximab therapy for Crohn's disease: a case report. J Crohns Colitis 2011;5:253255. doi: $10.1016 / j$.crohns.2010.12.007.
[19] Goujon C, Dahel K, Bérard F, Guillot I, Gunera-Saad N, Nicolas JF. Autoimmune hepatitis in two psoriasis patients treated with inflixmab. J Am Acad Dermatol 2010;63:e43-e44. doi: 10.1016/j.jaad.2009.02.029.

[20] Poulin Y, Thérien G. Drug-induced hepatitis and lupus during infliximab treatment for psoriasis: case report and literature review. J Cutan Med Surg 2010; 14:100-104. doi: 10.2310/7750.2009.09007.

[21] van Casteren-Messidoro C, Prins G, van Tilburg A, Zelinkova Z, Schouten J, de Man R. Autoimmune hepatitis following treatment with infliximab for inflammatory bowel disease. J Crohns Colitis 2012;6:630-631. doi: 10 . 1016/j.crohns.2012.01.017.

[22] Rodrigues S, Lopes S, Magro F, Cardoso H, Horta e Vale AM, Marques M, et al. Autoimmune hepatitis and anti-tumor necrosis factor alpha therapy: A single center report of 8 cases. World J Gastroenterol 2015;21:7584-7588. doi: 10.3748/wjg.v21.i24.7584.

[23] Dang LJ, Lubel JS, Gunatheesan S, Hosking P, Su J. Drug-induced lupus and autoimmune hepatitis secondary to infliximab for psoriasis. Australas J Dermatol 2014;55:75-79. doi: 10.1111/ajd.12054.

[24] Adar T, Mizrahi M, Pappo O, Scheiman-Elazary A, Shibolet O. Adalimumabinduced autoimmune hepatitis. J Clin Gastroenterol 2010;44:e20-e22. doi: 10.1097/MCG.0b013e3181a745e7.

[25] Cravo M, Silva R, Serrano M. Autoimmune hepatitis induced by infliximab in a patient with Crohn's disease with no relapse after switching to adalimumab. BioDrugs 2010;24 Suppl 1:25-27. doi: 10.2165/11586210-00000000000000.

[26] Goldfeld DA, Verna EC, Lefkowitch J, Swaminath A. Infliximab-induced autoimmune hepatitis with successful switch to adalimumab in a patient with Crohn's disease: the index case. Dig Dis Sci 2011;56:3386-3388. doi: 10. 1007/s10620-011-1748-1.

[27] Grasland A, Sterpu R, Boussoukaya S, Mahe I. Autoimmune hepatitis induced by adalimumab with successful switch to abatacept. Eur J Clin Pharmacol 2012;68:895-898. doi: 10.1007/s00228-011-1191-4.

[28] Harada K, Akai Y, Koyama S, Ikenaka Y, Saito Y. A case of autoimmune hepatitis exacerbated by the administration of etanercept in the patient with rheumatoid arthritis. Clin Rheumatol 2008;27:1063-1066. doi: 10.1007/ s10067-008-0885-1.

[29] Lis K, Kuzawińska O, Bałkowiec-Iskra E. Tumor necrosis factor inhibitors state of knowledge. Arch Med Sci 2014;10:1175-1185. doi: 10.5114/aoms. 2014.47827

[30] Bauer B, Chyou PH, Stratman EJ, Green C. Noninvasive testing for nonalcoholic steatohepatitis and hepatic fibrosis in patients with psoriasis receiving long-term methotrexate sodium therapy. JAMA Dermatol 2017. doi: 10. 1001/jamadermatol.2017.2083.

[31] Parakkal D, Sifuentes H, Semer R, Ehrenpreis ED. Hepatosplenic T-cell lymphoma in patients receiving TNF- $\alpha$ inhibitor therapy: expanding the groups at risk. Eur J Gastroenterol Hepatol 2011;23:1150-1156. doi: 10.1097/MEG. Ob013e32834bb90a.

[32] Keane J, Gershon S, Wise RP, Mirabile-Levens E, Kasznica J, Schwieterman $W D$, et al. Tuberculosis associated with infliximab, a tumor necrosis factor alpha-neutralizing agent. N Engl J Med 2001;345:1098-1104. doi: 10. 1056/NEJMoa011110.

[33] Lee SW, Park HJ, Kim BK, Han KH, Lee SK, Kim SU, et al. Leflunomide increases the risk of silent liver fibrosis in patients with rheumatoid arthritis receiving methotrexate. Arthritis Res Ther 2012;14:R232. doi: 10.1186/ar4075.

[34] Adiga A, Nugent K. Lupus hepatitis and autoimmune hepatitis (lupoid hepatitis). Am J Med Sci 2017;353:329-335. doi: 10.1016/j.amjms.2016.10. 014.

[35] Parekh R, Kaur N. Liver injury secondary to anti-TNF-alpha therapy in inflammatory bowel disease: A case series and review of the literature. Case Rep Gastrointest Med 2014;2014:956463. doi: 10.1155/2014/956463.

[36] Diamantis I, Boumpas DT. Autoimmune hepatitis: evolving concepts. Autoimmun Rev 2004;3:207-214. doi: 10.1016/j.autrev.2003.09.003.

[37] Vergani D, Mieli-Vergani G. Autoimmune hepatitis. Autoimmun Rev 2003;2: 241-247 doi: 10.1016/S1568-9972(03)00017-X.

[38] Teschke R, Schulze J, Eickhoff A, Danan G. Drug induced liver injury: Can biomarkers assist RUCAM in causality assessment? Int J Mol Sci 2017;18: E803. doi: 10.3390/ijms18040803.

[39] Eriksson C, Engstrand S, Sundqvist KG, Rantapää-Dahlqvist S. Autoantibody formation in patients with rheumatoid arthritis treated with anti-TNF alpha. Ann Rheum Dis 2005;64:403-407. doi: 10.1136/ard.2004.024182.

[40] Krawitt EL. Autoimmune hepatitis. N Engl J Med 2006;354:54-66. doi: 10. 1056/NEJMra050408. 